\title{
Exosomes derived from PC-3 cells suppress osteoclast differentiation by downregulating miR-148a and blocking the PI3K/AKT/mTOR pathway
}

\author{
GAOQIANG TIAN $^{1 *}$, KONGHE HU $^{1 *}$, SUJUN QIU ${ }^{2}$, YINGMING XIE ${ }^{2}$, \\ YANLIN CAO ${ }^{2}$, SONGJIA NI ${ }^{2}$ and LIFANG ZHANG ${ }^{3}$
}

\begin{abstract}
${ }^{1}$ Department of Spine Surgery, The Affiliated Yuebei People's Hospital of Shantou University Medical College, Shaoguan, Guangdong 512025; ${ }^{2}$ Department of Orthopedics, Zhujiang Hospital, The Second School of Clinical Medicine, Southern Medical University, Guangzhou, Guangdong 510280; ${ }^{3}$ Department of Oral and Maxillofacial Surgery, Stomatological Hospital of Southern Medical University, Guangzhou, Guangdong 510250, P.R. China
\end{abstract}

Received September 4, 2020; Accepted May 4, 2021

DOI: $10.3892 / \mathrm{etm} .2021 .10739$

\begin{abstract}
Prostate cancer is a leading malignancy in men that can also disrupt the bone tissue balance. Among all urological cancers, prostate cancer is associated with the highest rate of bone metastases, which can greatly reduce a patient's quality of life. In recent years, cell-derived exosomes, which can contain a wide range of biologically active molecules, have been reported as a novel method of communication among individual cells. However, the specific role that exosomes serve in this disease has not been fully elucidated. The prostate cancer cell line PC-3 were applied in the present study, where its exosomes were isolated to explore their potential effects on osteoclast differentiation. Exosomes are extracellular vesicles secreted by cells. The size of exosomes is $30-150 \mathrm{~nm}$. They have double membrane structure and saucer-like morphology. They contain rich contents (including nucleic acid, protein and lipid) and participate in molecular transmission between cells. The combined results of tartrate-resistant acid phosphatase staining (to identify osteoclasts obtained from human peripheral blood mononuclear cells), reverse transcription-quantitative PCR and western blotting showed that
\end{abstract}

Correspondence to: Dr Songjia Ni, Department of Orthopedics, Zhujiang Hospital, The Second School of Clinical Medicine, Southern Medical University, 253 Gongye Avenue, Guangzhou, Guangdong 510280, P.R. China

E-mail: warriorkof@163.com

Dr Lifang Zhang, Department of Oral and Maxillofacial Surgery, Stomatological Hospital of Southern Medical University, 366 Jiangnan Dadao South, Guangzhou, Guangdong 510250, P.R. China

E-mail: summere0615@163.com

*Contributed equally

Key words: osteoclast, exosome, prostate cancer, PC-3 cells, microRNA-148a
PC-3-derived exosomes attenuated osteoclast differentiation by downregulating marker genes associated with osteoclastic maturation, including V-maf musculoaponeurotic fibrosarcoma oncogene homolog B, matrix metalloproteinase 9 and integrin $\beta 3$. microRNA (miR)-148a expression was also found to be downregulated in osteoclasts by PC-3-derived exosomes. In addition, the mTOR and AKT signaling pathways were blocked after exposure to these PC-3 cell-derived exosomes. Therefore, results from the present study suggest that miR-148a mimics may be a new therapeutic approach for the prevention of prostate cancer bone metastases.

\section{Introduction}

Prostate cancer (PC) is becoming one of the leading male malignancies in a number of countries (1), which also has the highest incidence of bone metastases among all urological malignancies (2). The high probability of bone metastasis associated with prostate cancer places the patient at risk of pathological skeletal events, such as fractures (3). An osteolytic response is characterized by the destruction of normal bone due to osteoblast inactivation coupled with osteoclast recruitment and activation in the tumor-bone microenvironment (4). Osteolytic lesions are characterized by soft sections of damaged bone resulting from an osteolytic response that can cause bone pain and fractures (5).

Prostate cancer cells can produce substantial amounts of exosomes, which can contain important physiological and pathological information and can circulate around the human body in the blood or urine $(6,7)$. However, the specific role of $\mathrm{PC}$-derived-exosomes in the bone microenvironment remain poorly understood. Exosomes are extracellular vesicles with lipid bilayer membranes that are $30-150 \mathrm{~nm}$ in diameter and are secreted by cells. They have been reported to be involved in cell-cell communication, due to their ability to hold contain DNAs, mRNAs, microRNAs (miRNAs) and proteins $(8,9)$. In particular, miRNAs are molecules that can encode information that can be transferred among cells $(10,11)$. These findings provide evidence that exosomal miRNAs can be 
used as biomarkers for diagnosing various diseases, including cancer (12). Numerous tumor types, including prostate, lung and breast cancer, are particularly prone to bone metastasis and maintain substantial crosstalk with bone cells in the bone microenvironment (13). Colorectal cancer-secreted miR-25-3p can be transferred to HUVECs via exosomes (14). The promoting effect of exosomes on transendothelial migration in cancer was previously found to be positively correlated with miR-23a levels (15). However, it remains unknown which molecules contained within exosomes secreted by prostate cancer cells are involved in regulating bone homeostasis.

MicroRNAs (miRNAs) are small noncoding regulatory RNA molecules that are 22 nucleotides long and are encoded by plants, animals and certain types of viruses (16). MiRNAs are considered to be important regulators of bone metabolism and have differential expression profiles between patients with cancer and healthy individuals (17-19). According to a previous study, miRNAs remain relatively stable in clinical samples of plasma and serum (17). Circulating miRNAs identified in human primary osteoporosis, have also been implicated in supporting the progression of bone metastases in different tumors, such as PC, breast and non-small-cell lung cancer, esophageal squamous cell carcinoma and multiple myeloma, suggesting that these miRNAs can trigger the homing of cancer cells to the bone (19). In particular, miR-148a-3p was demonstrated to be significantly upregulated in osteoporotic human samples compared with non-osteoporotic human samples, where the increased miR-148a levels observed in the serum of patients with osteoporosis appears to be associated with the aforementioned miRNA upregulation observed in bone tissue of patients with osteoporosis (20). The functional role of miR-148a-3p in the bone suggests that this miRNA may serve as a viable biomarker for postmenopausal osteoporosis $(21,22)$. Furthermore, exosomal miR-148a has been reported to serve a positive regulatory role in osteoclast differentiation, where V-maf musculoaponeurotic fibrosarcoma oncogene homolog B (MafB) was found to be the target of miR-148a in osteoclast differentiation (23). Therefore, MafB was considered to be the marker of osteoclastic differentiation in the present study.

The PI3K/AKT/mTOR signaling pathway has been documented to regulate the migration and invasion of prostate cancer, which is also mentioned frequently as the signaling pathway that can be explored in other malignant tumors, such as breast cancer and B-cell lymphoproliferative disorders (24,25). Although previous findings found targeting this pathway to be an option the prevention and treatment of bone metastasis caused by prostate cancer (26-28), the potential connection between PC-derived exosomes and the $\mathrm{PI} 3 \mathrm{~K} / \mathrm{AKT} / \mathrm{mTOR}$ signaling pathway remain unclear.

In the present study, the possible function of exosomes derived from prostate cancer on the bone microenvironment and MafB expression in osteoclasts was examined. Exosomes isolated from the prostate cancer cell line PC-3 were cocultured with pre-osteoclast cells from volunteers. The present study investigated the inhibitory effect of PC-3-derived exosomes on osteoblast differentiation, which was detected by western blotting and reverse transcription-quantitative (RT-q)PCR.

\section{Materials and methods}

PC-3 cell culture. The human prostate cancer epithelial cell line PC-3 (cat. no. CRL-1435; American Type Culture Collection) was used in this study. PC-3 cells were cultured in RPMI-1640 (Gibco; Thermo Fisher Scientific, Inc.) supplemented with $10 \%$ FBS (Biological Industries), $100 \mathrm{U} / \mathrm{ml}$ penicillin and $100 \mu \mathrm{g} / \mathrm{ml}$ streptomycin (Gibco; Thermo Fisher Scientific, Inc.) and incubated at $37^{\circ} \mathrm{C}$ in $5 \% \mathrm{CO}_{2}$. The cell culture medium was changed every 2-3 days. At $80 \%$ confluence, the cell culture medium was replaced by RPMI-1640 medium with exosome-depleted serum (Thermo Fisher Scientific, Inc.) for $48 \mathrm{~h}$ at $37^{\circ} \mathrm{C}$ before the cell culture medium was collected for exosome isolation.

Exosome isolation. The current 'gold standard' for the purification of a subset of exosomes is differential centrifugation, which typically consists of low-speed centrifugation to remove cells and large vesicles and high-speed ultracentrifugation to pellet exosomes (29). To isolate exosomes, the cell culture medium (CCM) were collected. Briefly, any detached and dead cells were removed from the CCM by serial centrifugation at $300 \mathrm{x} \mathrm{g}$ for $10 \mathrm{~min}$ at $4^{\circ} \mathrm{C}$ and $2,000 \mathrm{x} \mathrm{g}$ for $20 \mathrm{~min}$ at $4^{\circ} \mathrm{C}$. A further centrifugation procedure was performed at $10,000 \times \mathrm{g}$ at $4^{\circ} \mathrm{C}$ for $30 \mathrm{~min}$ to remove any cell debris. Supernatants were then collected and filtered through $0.22 \mathrm{~mm}$ filters (Merck KGaA) to remove any contaminating apoptotic bodies, microvesicles and cell debris. The clarified CCM was then centrifuged in a Beckman Coulter Optima ${ }^{\mathrm{TM}} \mathrm{L}-80 \mathrm{XP}$ Ultracentrifuge at $100,000 \mathrm{xg}$ at $4^{\circ} \mathrm{C}$ for $70 \mathrm{~min}$ with a Type $32.8 \mathrm{Ti}$ rotor to pellet the exosomes. The supernatant was carefully removed and the resulting exosome pellet was resuspended in $100 \mu \mathrm{l}$ PBS. Protein concentration in the exosomes was determined by using a bicinchoninic protein assay.

Exosome characterization. According to the manufacturer's guidelines, we determined the number and size distribution of the exosomes with a NanoSight LM10 (Malvern Panalytical). In total, $1 \mathrm{ml}$ sample was injected into the sample chamber using a sterile syringe. After that, the morphology of exosomes was observed by transmission electron microscopy (TEM). Sample $(1: 1,000)$ was dropped onto a carbon-coated 200-mesh copper grid for a 1-min incubation in $2.5 \%$ glutaraldehyde fixing solution at room temperature. Any extra liquid was absorbed gently using a filter paper around the border of the grids. The sample was stained with $2 \%$ aqueous solution of phosphotungstic acid for $30 \mathrm{sec}$ at room temperature. Extra liquid was absorbed again by filter paper. The grids were examined using an H-7650 TEM (Hitachi, Ltd.) at $80 \mathrm{kV}$. After the sample was heated for $1 \mathrm{~min}$ at room temperature and embedded in epoxy resin at $37^{\circ} \mathrm{C}$ for $12 \mathrm{~h}$, particle morphology was observed. Additionally, nanoparticle tracking analysis was performed to assess the size distribution of PC-3 exosomes using the NanoSight LM10 (Malvern Panalytical).

Furthermore, the expression of exosome markers was measured by flow cytometry using an Accuri C6 flow cytometer (BD Biosciences).

Exosome labeling. PKH fluorescent dyes, which label cell membranes by the insertion of their aliphatic chains into the lipid bilayer, have been widely used to label exosomes based 
on their intense signal and long half-life (30,31). PC-3-derived exosomes were labeled with PKH26 according to the manufacturer's protocol (Umibio Science and Technology Group; cat. no. UR52302). Briefly, the fluorescent stain solution contained $9 \mu \mathrm{l}$ Diluent $\mathrm{C}$ and $1 \mu \mathrm{l}$ PKH26. The exosome suspension made as aforementioned was mixed with the stain solution and incubated for $10 \mathrm{~min}$ at $37^{\circ} \mathrm{C}$ protected from light. After coculturing exosome $\left(50 \mathrm{ng} / 1 \times 10^{3}\right.$ cells $)$ with preosteoclasts $\left(1 \times 10^{5}\right)$ at $37^{\circ} \mathrm{C}$ for 12 days, the culture media were removed before the cells were fixed with $4 \%$ paraformaldehyde at room temperature for $5 \mathrm{~min}$ and washed with PBS three times. The cells were then blocked with 5\% BSA (Beyotime Institute of Biotechnology) for $10 \mathrm{~min}$ at room temperature and the nuclei were stained with $5 \mu \mathrm{g} / \mathrm{ml}$ DAPI for $5 \mathrm{~min}$ at room temperature. Images of the cells were acquired using a fluorescence microscope (x100; magnification; Leica-DMi8; Leica Microsystems $\mathrm{GmbH}$ ).

Human osteoclast induction. To induce osteoclasts, human peripheral blood mononuclear cells (PBMCs) were obtained from the peripheral blood of six healthy male donors (age, 25-29 years; mean age, 26.7 years) who have signed informed consent forms on August 10, 2019, in Zhujiang Hospital Outpatient Blood Sampling Center (Guangzhou, China), where the samples were collected into centrifuge tubes with $1,000 \mathrm{U} / \mathrm{ml}$ heparin under a protocol approved by the Committee of Clinical Ethics of Zhujiang Hospital (Guangzhou, China). The human peripheral blood samples were diluted with an equal volume of PBS and centrifuged in a centrifuge tube containing the same volume of Histopaque-1077 (Sigma-Aldrich; Merck KGaA) at $500 \mathrm{x}$ g for $30 \mathrm{~min}$ at $25^{\circ} \mathrm{C}$ with slow deceleration. After centrifugation, PBMCs located at the interface between the plasma and Histopaque-1077 were collected and washed with PBS twice, followed by centrifugation at $250 \mathrm{x} \mathrm{g}$ for $10 \mathrm{~min}$ at $25^{\circ} \mathrm{C}$. The isolated PBMCs were suspended and incubated in complete RPMI-1640 medium containing $50 \mathrm{ng} / \mathrm{ml}$ macrophage colony-stimulating factor (M-CSF; Sino Biological, Inc.) in six-well plates at a density of $1 \times 10^{6}$ cells $/ \mathrm{ml}$ per well in $5 \% \mathrm{CO}_{2}$ at $37^{\circ} \mathrm{C}$ for 3 days. Any non-adherent cells were then washed away with PBS to obtain adherent mononuclear cells. To stimulate osteoclast differentiation, $100 \mathrm{ng} / \mathrm{ml}$ receptor activator of nuclear factor $\kappa \mathrm{B}$ ligand (RANKL; Sino Biological, Inc.) was added to complete exosome-free serum RPMI-1640 medium after removing non-adherent cells in the absence (control) or presence of PC-3 exosomes at three different concentrations (10, 30 and $50 \mathrm{ng}$ per $1 \times 10^{3}$ cells seeded) in $5 \% \mathrm{CO}_{2}$ at $37^{\circ} \mathrm{C}$ for 12 days. The culture medium was replaced every 3 days with fresh media supplemented with the aforementioned reagents [RPMI-1640 containing $50 \mathrm{ng} / \mathrm{ml}$ macrophage colony-stimulating factor and $100 \mathrm{ng} / \mathrm{ml}$ receptor activator of nuclear factor- $\kappa \mathrm{B}$ ligand and different concentrations of PC-3 exosomes (10, 30 and 50 ng per $1 \times 10^{3}$ cells seeded)]. Tartrate-resistant acid phosphatase (TRAP) staining was used for osteoclast staining whereas western blotting and reverse transcription-quantitative PCR were used to demonstrate how osteoclast differentiation changed among these concentrations of PC-3 exosomes.

TRAP staining. TRAP staining was used to confirm the shape of osteoclasts according to the manufacturer's protocol (Sigma
Aldrich; Merck KGaA). CCM was first removed before the osteoclasts $\left(1 \times 10^{5}\right)$ in six-well plates were washed twice with PBS. Osteoclasts were fixed with Fixative Solution (a combination of $25 \mathrm{ml}$ citrate solution, $65 \mathrm{ml}$ acetone and $8 \mathrm{ml} \mathrm{37 \%}$ formaldehyde) for $30 \mathrm{sec}$ at room temperature. The samples were then rinsed with deionized water three times. At the same time, $0.5 \mathrm{ml}$ Fast Garnet GBC base solution and $0.5 \mathrm{ml}$ sodium nitrite solution were mixed for $30 \mathrm{sec}$ and added into a $10-\mathrm{ml}$ beaker containing $0.5 \mathrm{ml}$ naphthol AS-BI phosphate solution, $2 \mathrm{ml}$ acetate solution and $1 \mathrm{ml}$ tartrate solution. Next, the osteoclasts were immersed in this mixture, incubated at $37^{\circ} \mathrm{C}$ for $1 \mathrm{~h}$ and rinsed with distilled water three times. TRAP-positive cells appeared red or purple, where each of them had $\geq$ three nuclei.

miRNA mimic/inhibitor transfection. miR-148a mimics, miR-148a inhibitor and corresponding negative controls (NC) were purchased from Shanghai GenePharma Co., Ltd. Their sequences were as follows: miR-148a mimic sense, 5'-UCA GUGCACUACAGAACUUUGU-3' and anti-sense, 5'AAA GUUCUGUAGUGCACUGAUU-3'; miR-148a inhibitor, 5'-ACAAAGUUCUGUAGUGCACUGA-3'; inhibitor NC, 5'-CAGUACUUUUGUGUAGUACAA-3' and mimic NC, 5'-UUGUAC UACACA AAAGUACUG-3'. All NCs are non-targeting sequences. Cells transfected with the mimic NC and inhibitor NC are designated as control groups. PBMCs were transfected with $5 \mu \mathrm{lmiR}-148 \mathrm{a}$ mimic, inhibitor, mimic $\mathrm{NC}$ or inhibitor NC by using the RFect siRNA transfection reagent (cat. no. BIOG-11014; Changzhou EMI Biotechnology Co.,Ltd.) in six-well plates when cells reached $80 \%$ confluence. After transfection for $6 \mathrm{~h}$, the PBMCs were induced to undergo osteoclast differentiation by M-CSF + RANKL treatment in $5 \% \mathrm{CO}_{2}$ at $37^{\circ} \mathrm{C}$ for 12 days with the medium composition as aforementioned.

Total RNA Extraction and RT-qPCR. RNA was isolated by TRIzol ${ }^{\circledR}$ extraction (Thermo Fisher Scientific, Inc.) and reverse-transcribed using an Evo M-MLV RT kit with gDNA Clean for $\mathrm{qPCR}$ at $42^{\circ} \mathrm{C}$ for $2 \mathrm{~min}$ and stored at $4^{\circ} \mathrm{C}$ (Accurate Biotechnology Co., Ltd.; cat. no. AG11705). Primers for qPCR were designed by Sangon Biotech Co.,Ltd. as follows: miR-148a forward, 5'-GCGCGTCAGTGCACTACAGAA-3' and reverse, 5'-AGTGCAGGGTCCGAGGTATT-3'; U6 forward, 5'-AGA GAAGATTAGCATGGCCCCTG-3' and reverse, 5'-AGT GCAGGGTCCGAGGTATT-3'; MafB forward, 5'-AGAAGC GGCGGACCCTGAAG-3' and reverse, 5'-GCTGCTCCA CCTGCTGAATGAG-3'; MMP-9 forward, 5'-TCCTCTTAT GCCTGCCTGTCTCC-3' and reverse, 5'-CTTGGTCCACCT GGTTCAACTCAC-3' and integrin $\beta 3$ (I $\beta 3$ ) forward, 5'-GTG ACCTGAAGGAGAATCTGC-3' and reverse, 5'-CCGGAG TGCAATCCTCTGG-3'. U6 and GAPDH (forward, 5'-CCG CATCTTCTTTTGCGTCG-3' and reverse, 5'-CCCAATACG ACCAAATCCGTTG-3') were used as internal references. qPCR was performed in triplicate using the SYBR Green Premix Pro Taq HD qPCR kit (cat. no. AG11721; Accurate Biotechnology Co., Ltd.). PCRs were run and analyzed using the Applied Biosystems StepOnePlus ${ }^{\text {TM }}$ Real-Time PCR System (Thermo Fisher Scientific, Inc.). The reaction conditions were pre-heating at $95^{\circ} \mathrm{C}$ for $3 \mathrm{~min}$, followed by 40 cycles of denaturation at $95^{\circ} \mathrm{C}$ for $30 \mathrm{sec}$ and annealing at $60^{\circ} \mathrm{C}$ for $30 \mathrm{sec}$. Gene expression was represented as 

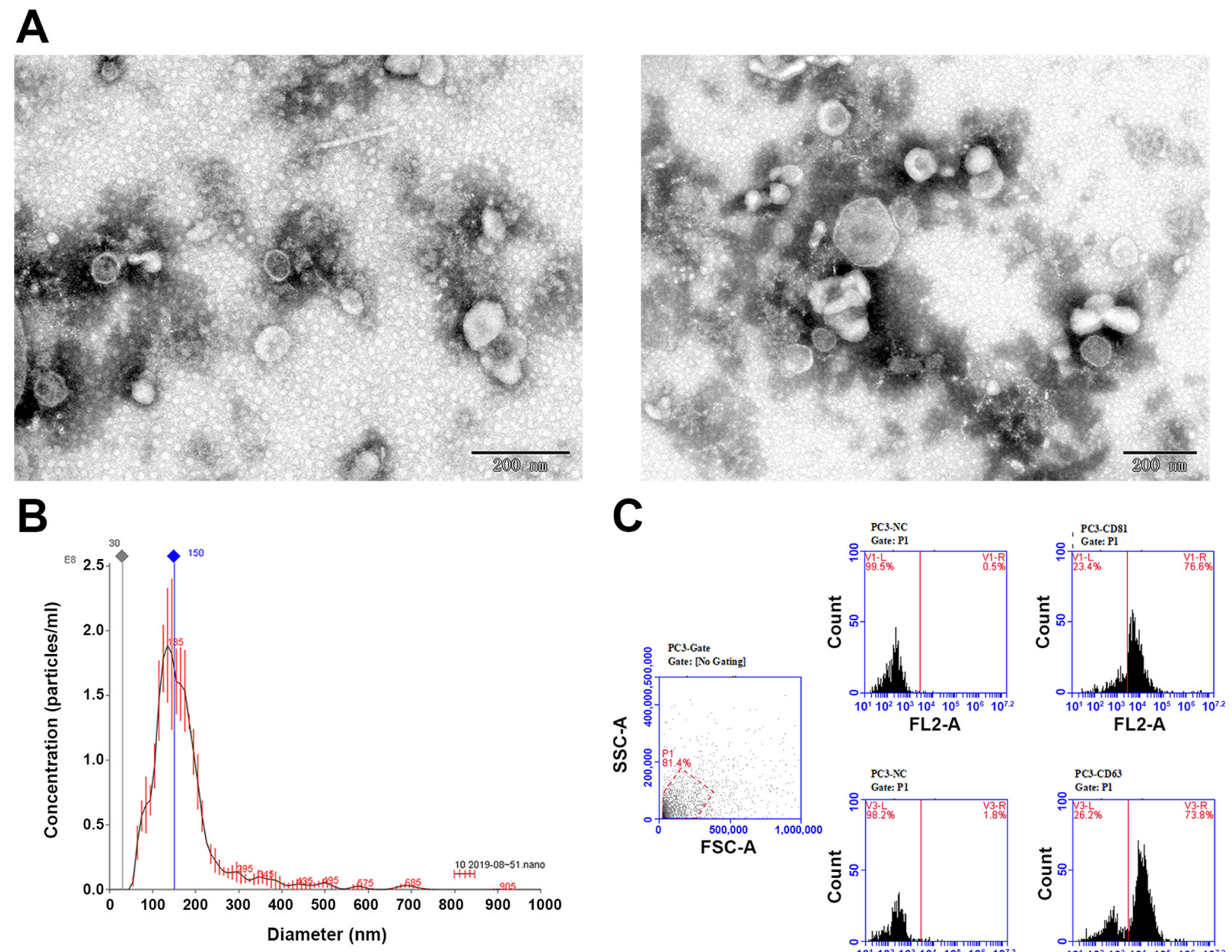

Selected concentration: 0.84 E9 particles/ml (38.3\%)
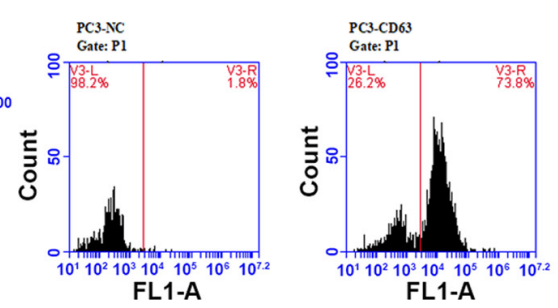

D

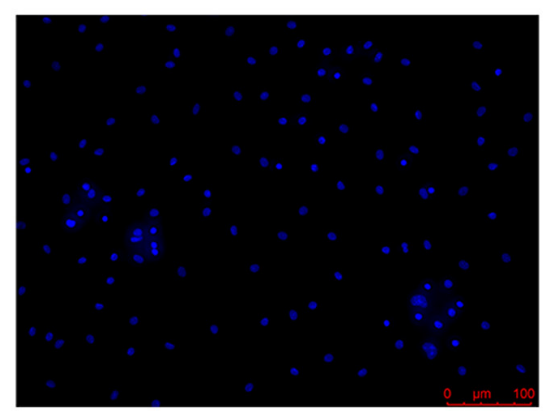

PKH26

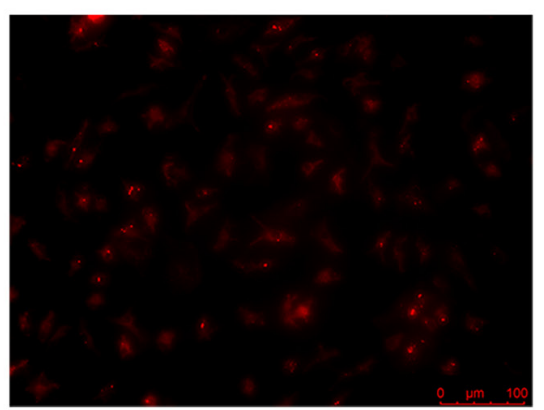

Merged

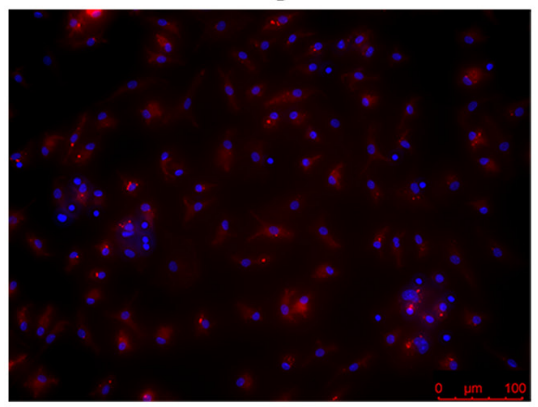

Figure 1. Characterization of PC-3-derived exosomes. (A) Electron microscopic images of exosomes isolated from PC-3 cells. Scale bars, 200 nm. (B) Nanoparticle tracking analysis of the isolated exosomes. (C) Flow cytometric analysis of the expression of exosome surface markers CD63 and CD81. (D) Uptake of PC-3-derived exosomes by preosteoclasts. Red, PKH26-labeled exosomes; blue: DAPI-stained nuclei. Scale bars, $100 \mu$ m. NC, negative control.

$\Delta \mathrm{Cq}=\mathrm{Cq}_{\text {gene }}-\mathrm{Ct}_{\text {reference }}$, and the multiplication ratio was obtained using the $2^{-\Delta \Delta \mathrm{Cq}}$ method (32).

Western Blotting. Osteoclasts were lysed in RIPA lysis buffer (CoWin Biosciences) containing phosphatase (CoWin Biosciences) and protease inhibitors (CoWin Biosciences). The cell lysates were centrifuged at $12,000 \mathrm{x}$ g at $4^{\circ} \mathrm{C}$ for $15 \mathrm{~min}$ and the protein concentration collected from the supernatant was determined using a bicinchoninic acid Protein Assay kit. $60 \mu \mathrm{g}$ of protein samples $(80 \mu \mathrm{g})$ were separated by $8 \%$ SDS-PAGE and then transferred onto PVDF membranes. The membranes were blocked in 5\% nonfat dry milk diluted in tris-buffered saline containing $0.1 \%$ Tween 20 (TBST) at room temperature for $1 \mathrm{~h}$. Afterwards, the membranes were incubated with primary rabbit monoclonal antibodies against the following antigens at $4^{\circ} \mathrm{C}$ overnight: MafB $(1: 1,000$; Zen-bio; cat. no. 862796), MMP-9 (1:1,000; Abcam; \#EP1254), I $\beta 3$ (1:1,000; Cell Signaling Technology, Inc.), AKT, phosphorylated (p)-AKT, mTOR, p-mTOR and GAPDH (all 1:2,000; all Wuhan Boster Biological Technology, Ltd.). After washing, the membranes were incubated with goat anti-rabbit IgG secondary antibody (cat. no. BA1040, 1:10,000; Wuhan Boster 


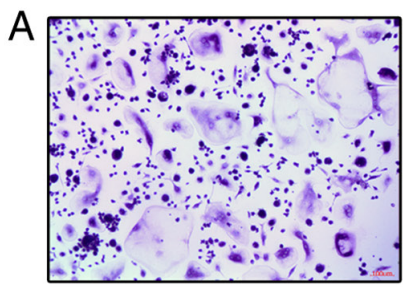

B
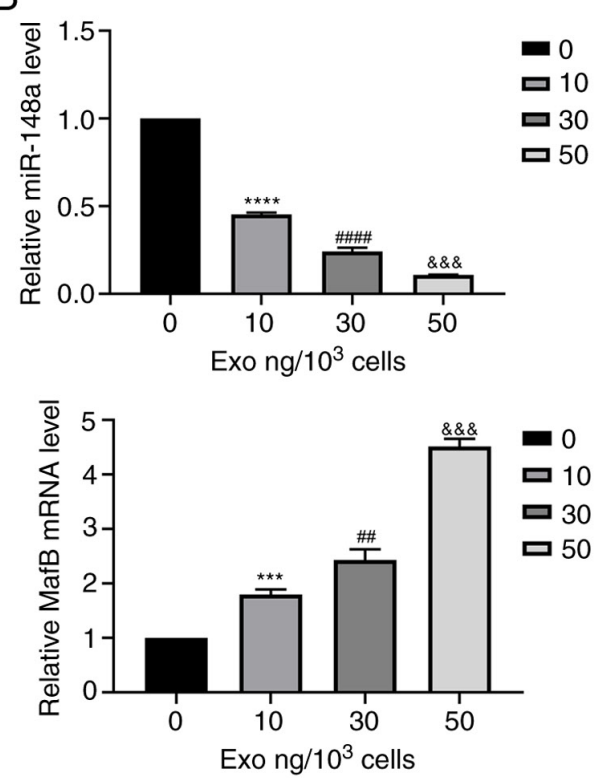
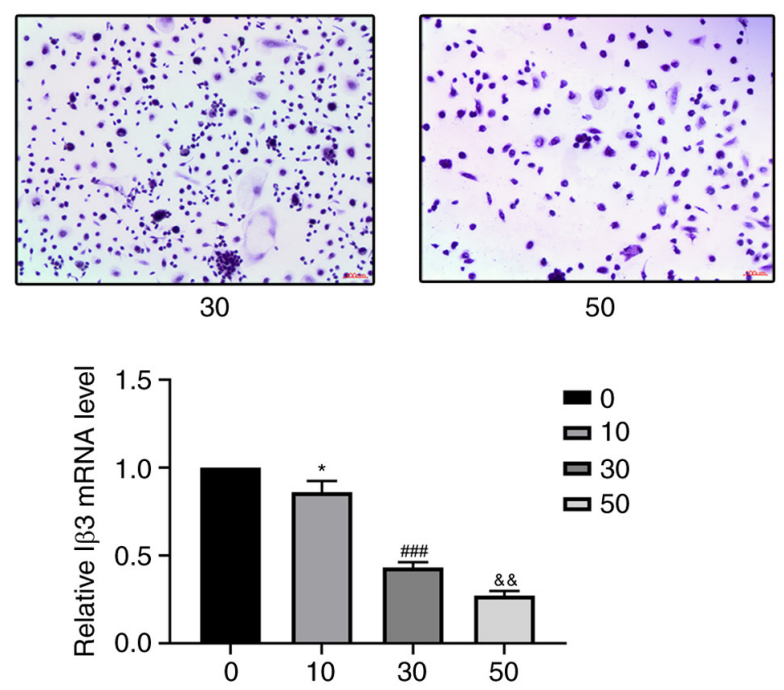

च 0
口 10
口 30
口 50
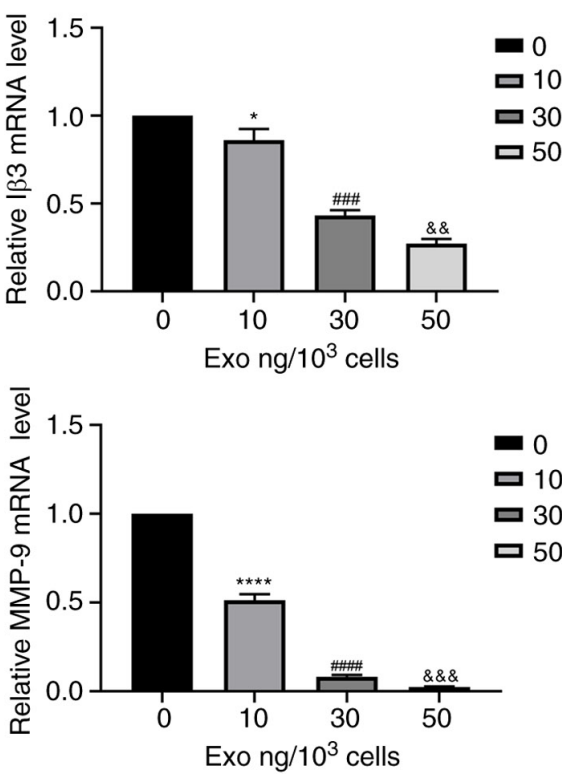

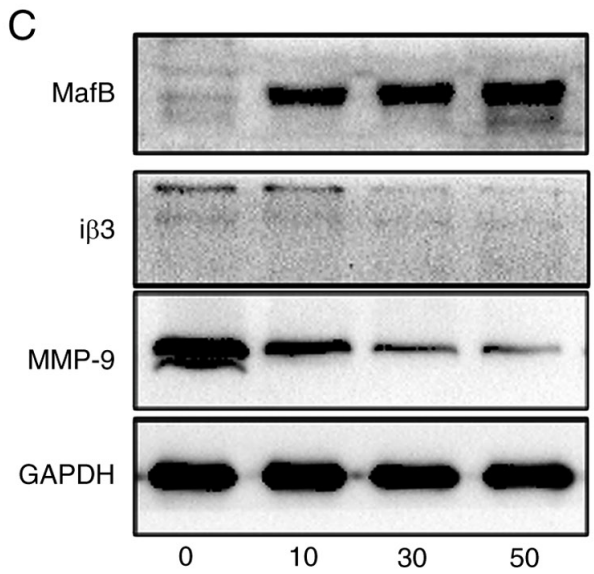

Figure 2. PC-3-derived exosomes inhibit osteoclast differentiation. (A) Tartrate-resistant acid phosphatase staining of osteoclasts treated with 0 , 10, 30 or $50 \mathrm{ng}$ exosomes $/ 1,000$ cells. Scale bar, $100 \mu \mathrm{m}$. (B) Relative expression levels of miR-148a, MafB, MMP-9 and I 33 in osteoclasts treated with 0, 10,30 , or $50 \mathrm{ng}$ exosomes/1,000 cells. (C) Western blotting of MafB, MMP-9 and I $\beta 3$ in osteoclasts after treatment with 0, 10,30 or 50 ng exosomes/1,000 cells

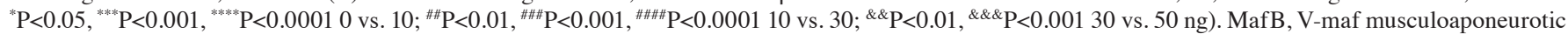
fibrosarcoma oncogene homolog B; MMP, matrix metalloproteinase; $\mathrm{I} \beta 3$, integrin $\beta 3$; miR, microRNA; exo, exosome.

Biological Technology, Ltd.) at room temperature for $1 \mathrm{~h}$ and washed with TBST three times. Proteins were visualized using a Tanon 4200 SF automated fluorescence chemiluminescence image analysis system (Tanon Science and Technology Co., Ltd.) using an ECL kit (KeyGEN Biotech; cat. no. KGP1121). The image lab software (v3.0; Bio-Rad Laboratories, Inc.) was used to analyze the results of western blotting.

Statistical Analysis. All data are presented as the mean \pm standard deviations. Comparisons were made using unpaired Student's t-test and one-way ANOVA followed by the Tukey's post hoc test with GraphPad Prism version 8.0 (GraphPad Software, Inc.). $\mathrm{P}<0.05$ was considered to indicate a statistically significant difference. All experiments were repeated $\geq$ three times.

\section{Results}

Characteristics of $P C-3$ Exosomes. To investigate the specific effect of the exosomes from PC-3 cells on osteoclast differentiation, ultracentrifugation was used to collect exosomes from the PC-3 prostate cancer cells that were incubated 


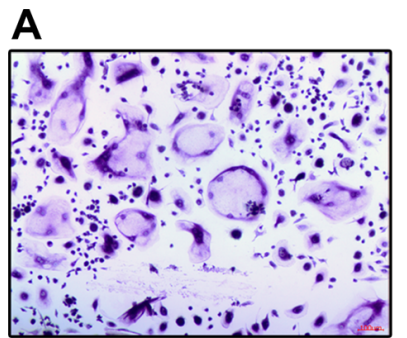

Mimic

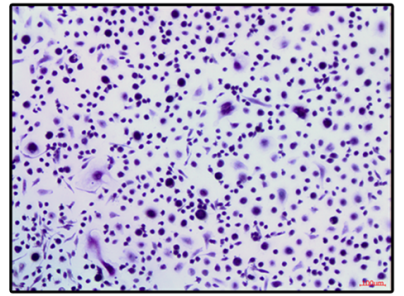

Mimic NC

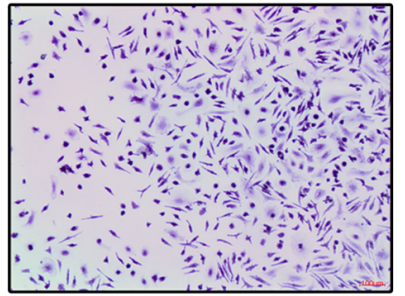

Inhibitor

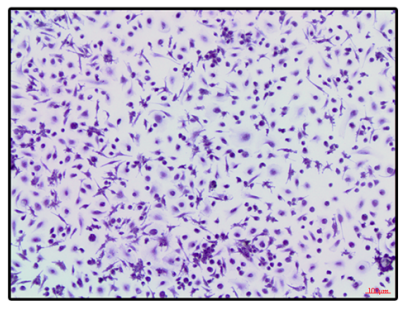

Inhibitor NC
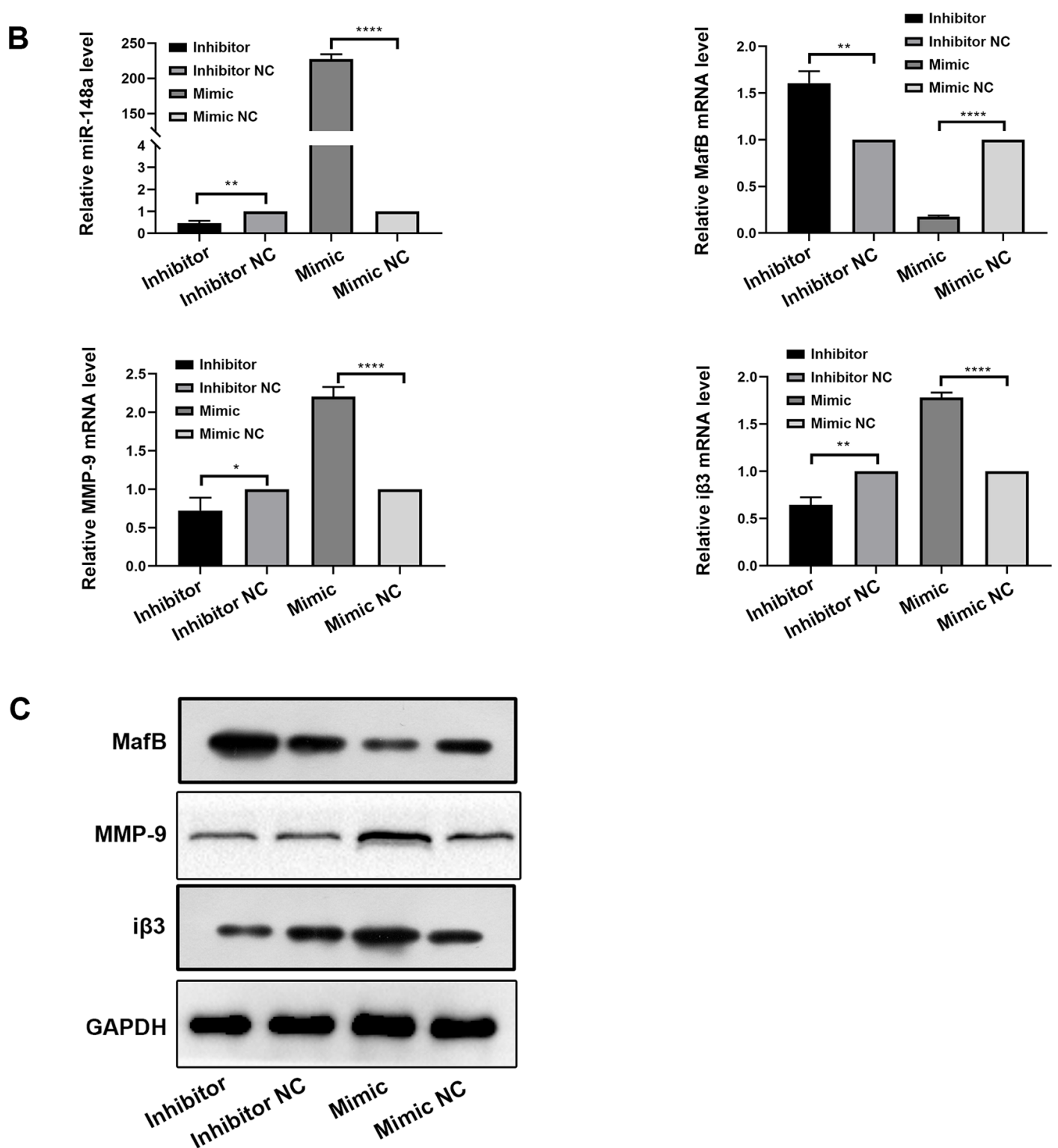

Figure 3. miR-148a downregulation inhibits osteoclast differentiation. (A) Tartrate-resistant acid phosphatase staining in osteoclasts expressing the miR-148a mimic, mimic NC, inhibitor or inhibitor NC after induction with M-CSF and RANKL. Scale bar, $100 \mu \mathrm{m}$. (B) Relative miR-148a, MafB, MMP-9 and I 33 expression in osteoclasts transfected with miR-148a mimic, inhibitor, mimic NC or inhibitor NC after induction with M-CSF and RANKL was detected by reverse transcription-quantitative PCR. (C) Western blotting of MafB, MMP-9 and I 33 in osteoclasts transfected with miR-148a mimic, inhibitor, mimic NC or inhibitor NC after induction with M-CSF and RANKL. ${ }^{*} \mathrm{P}<0.05,{ }^{* * *} \mathrm{P}<0.01,{ }^{* * * * * *} \mathrm{P}<0.0001$. MafB, V-maf musculoaponeurotic fibrosarcoma oncogene homolog B; MMP, matrix metalloproteinase; I $\beta 3$, integrin $\beta 3$; miR, microRNA; M-CSF, macrophage colony-stimulating factor; RANKL, receptor activator of nuclear factor $\kappa \mathrm{B}$ ligand; $\mathrm{NC}$, negative control.

with exosome-free serum. The exosomes were examined by TEM and nanoparticle tracking analysis. According to the TEM results, the isolated exosomes appeared as round or oval membrane vesicles (Fig. 1A) with sizes within the characteristic diameter range of $45-150 \mathrm{~nm}$. The average size of the exosomes from PC-3 cells was $176.5 \mathrm{~nm}$ and the peak diameter was $136 \mathrm{~nm}$ (Fig. 1B). These exosomes were verified further by detecting the exosome-specific markers CD81 and 
A
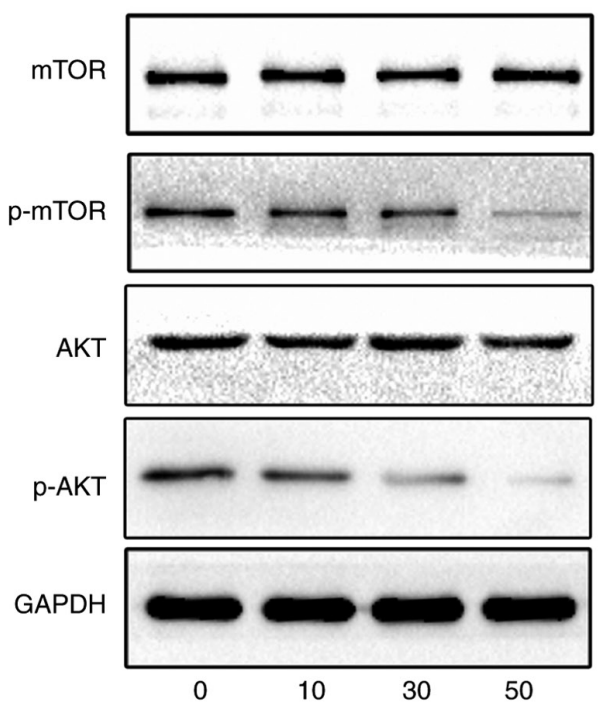

C
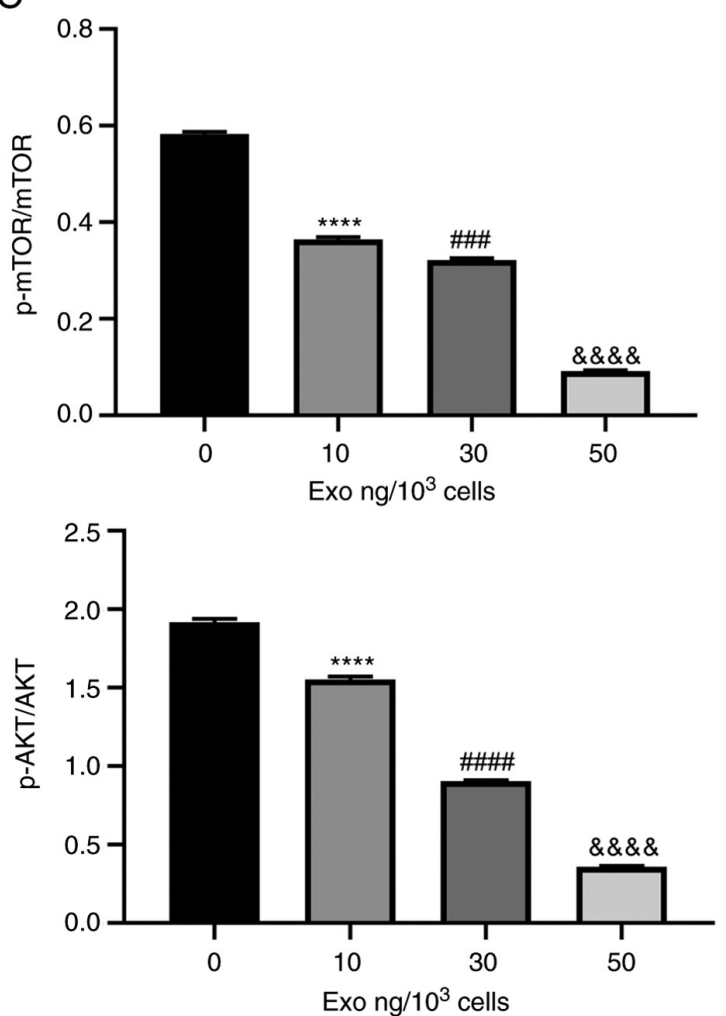

B
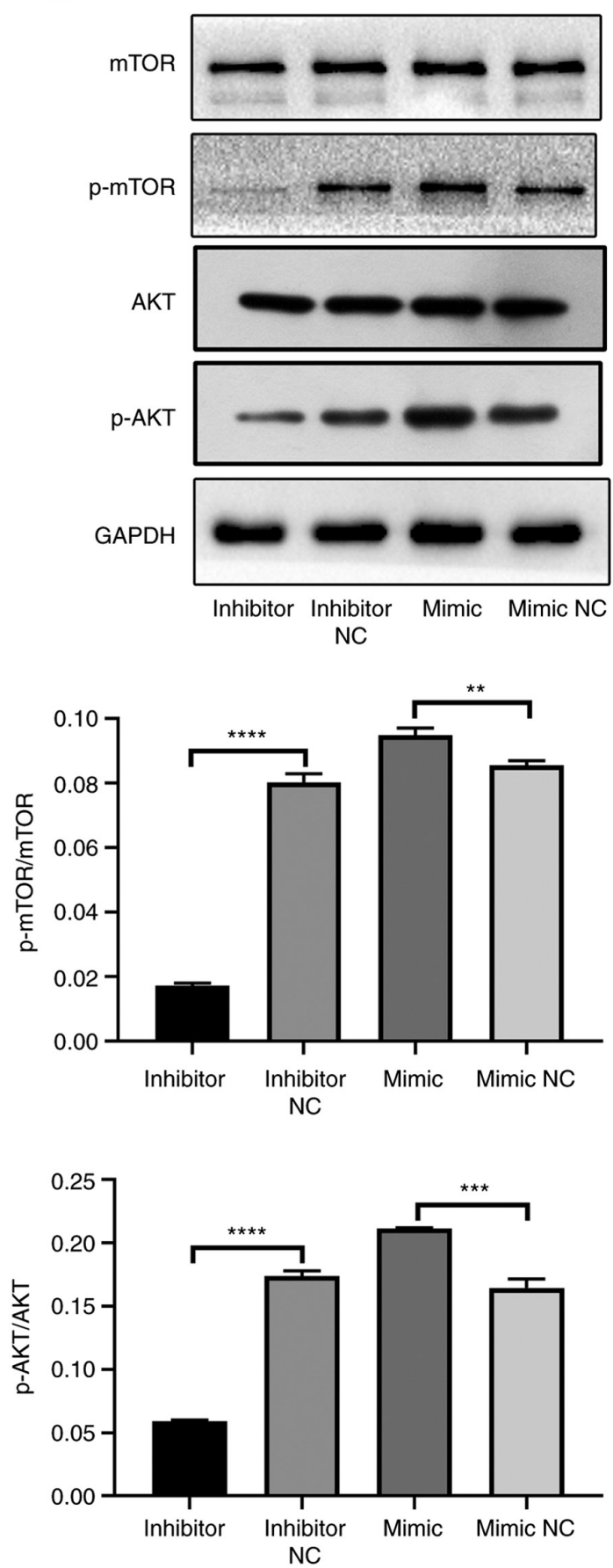

Figure 4. PC-3-derived exosomes block the AKT and mTOR pathways by miR-148a downregulation. (A) Western blotting of p-AKT, AKT, p-mTOR and mTOR in osteoclasts treated with $0,10,30$ or 50 ng exosomes/1,000 cells. (B) Western blotting of p-AKT, AKT, p-mTOR and mTOR in osteoclasts transfected with miR-148a mimic, inhibitor, mimic NC or inhibitor NC after induction with M-CSF and RANKL. (C) Semi-quantification of western blot analysis of p-AKT/AKT and p-mTOR/mTOR. ${ }^{* *} \mathrm{P}<0.01,{ }^{* * *} \mathrm{P}<0.001,{ }^{* * * * *} \mathrm{P}<0.00010$ vs. 10 ; ${ }^{\# \# \#} \mathrm{P}<0.001,{ }^{\# \# \# \#} \mathrm{P}<0.000110$ vs. 30; \&\&\&\&P<0.0001 30 vs. 50 ng. p-, phosphorylation; miR, microRNA; NC, negative control; M-CSF, macrophage colony-stimulating factor; RANKL, receptor activator of nuclear factor $\kappa \mathrm{B}$ ligand.

CD63 by flow cytometry, which were expressed at 76.6 and $73.8 \%$, respectively (Fig. 1C).

Pre-osteoclasts uptake PC-3-derived exosomes. The present study then investigated if the PC-3-derived exosomes can be taken up by the pre-osteoclasts. The exosomes were first labeled with the fluorescent dye PKH26 and then added into the culture medium of preosteoclasts. After $12 \mathrm{~h}$, the preosteoclasts exhibited efficient uptake of the PC-3-derived exosomes, as indicated by the presence of red fluorescence staining in these cells (Fig. 1D).

PC-3-derived exosomes inhibit osteoclast differentiation. According to the results of TRAP staining, marked differences in osteoclast differentiation were found among the groups of PBMCs exposed to three different concentrations of PC-3 exosomes after coculturing for 10 days with M-CSF and RANKL (Fig. 2A). The differentiation of osteoclasts 
decreased when the concentration of exosomes increased. The most notable inhibition was observed in the group treated with $50 \mathrm{ng} / 1,000$ cells (Fig. 2A). It was also found that PC-3-derived exosomes reduced the expression level of miR-148a and inhibited the differentiation of osteoclasts by decreasing the protein and mRNA expression of osteoclastic markers, including MMP-9 and I $\beta-3$ and increasing the protein and mRNA expression of $\mathrm{V}$-maf musculoaponeurotic fibrosarcoma oncogene homolog B (MafB) (Fig. 2B and C).

Downregulation of miR-148a inhibits the differentiation of osteoclasts. To further investigate if miR-148a serves a role during osteoclast differentiation, PBMCs were transfected with miR-148a mimic, miR-148a inhibitor, mimic NC or inhibitor NC. First, osteoclasts were subjected to TRAP staining (Fig. 3A). Transfection with the miR-148a mimic increased the differentiation of osteoclasts and formed more large particles of purplish red positive staining cells compared with that the mimic NC group, whilst the miR-148a inhibitor reduced osteoclast differentiation compared with that in the inhibitor NC group. The expression level of miR-148a and osteoclastic markers MafB, MMP-9, I $\beta 3$ were also measured by RT-qPCR among the four groups, where miR-148a, MMP-9 and $\mathrm{I} \beta 3$ expression in the mimic group were significantly higher compared with that in the mimic NC group, whilst expression in the inhibitor group were significantly lower compared with that in the inhibitor NC group (Fig. 3B). MafB expression levels in the mimic group were lower than in the mimic NC group, and higher in the inhibitor group compared with inhibitor NC. From western blotting, MafB expression was markedly upregulated, whereas MMP-9 and I 33 expression were markedly downregulated after transfection with the miR-148a inhibitor compared with those in osteoclasts transfected with inhibitor NC (Fig. 3C). By contrast, transfection with the miR-148a mimics resulted in the opposite effects compared with the observations in the miR-148a inhibitor groups (Fig. 3B and C). Therefore, inhibiting the expression of miR-148a in PBMCs attenuated differentiation into osteoclasts, whilst upregulating the expression of miR-148a promoted differentiation into osteoclasts.

PC-3-derived exosome downregulates the AKT and mTOR pathways. To further investigate the underlying mechanism, activities of the AKT and mTOR signaling pathways were focused upon. The protein levels of AKT, p-AKT, p-mTOR and mTOR were first measured in osteoclasts cultured with various concentrations of PC-3-derived exosomes. The phosphorylation of AKT and mTOR were significantly suppressed by PC-3-derived exosomes in a concentration-dependent manner (Fig. 4A). However, the total AKT and mTOR levels remain unchanged. Subsequently, the effect of miR-148a on the AKT and mTOR signaling pathways were investigated. The levels of p-mTOR, mTOR, p-AKT, and AKT were measured in the miR-148a mimic, inhibitor, mimic NC and inhibitor NC groups. The levels of AKT and mTOR phosphorylation were significantly increased in the miR-148a mimic group compared with those in the mimic NC group (Fig. 4B). By contrast, their levels were significantly lower in the miR-148a inhibitor group compared with those in the inhibitor NC group (Fig. 4B). Furthermore, miR-148a overexpression and inhibition exerted no effects on total mTOR and AKT expression levels (Fig. 4B). These results suggest that PC-3-derived exosomes can block the mTOR and AKT signaling pathways by miR-148a downregulation.

\section{Discussion}

In the present study, the results suggest that exosomes from the PC-3 prostate cancer cell line suppressed the differentiation of osteoclasts. The osteoclastic maturation markers I $\beta 3$ and MMP-9 were found to be attenuated while MafB was upregulated, where miR-148a expression was also downregulated. In addition, the mTOR and AKT signaling pathways were suppressed by exosomes from PC-3 cells. Combining these results and the previous findings that osteoblastic bone metastases in prostate cancer are caused by tumor-derived factors, which lead to osteoblast proliferation, differentiation and bone formation (33), suggest that prostate cancer bone metastases are mainly osteoblastic (34). The present study explored whether miR-148a has a role in prostate cancer bone metastases. It was shown that upregulating miR-148a expression promoted differentiation of osteoclasts, whilst downregulation of miR-148a suppressed osteoclast differentiation, thus this may be a novel treatment method.

Torrealba et al previously suggested that the $\mathrm{PI} 3 \mathrm{~K} / \mathrm{AKT} / \mathrm{mTOR}$ pathway is an important therapeutic target and a viable option as a predictive biomarker for the onset, behavior and progression of PC after prostatectomy (35). Therefore, the present study mainly aimed to investigate activities of the mTOR and AKT pathways following treatment with PC-3-derived exosomes in prostate cancer bone metastases. Reduced expression of miR-148a inhibited the mTOR and AKT pathways in the present study. It is therefore possible that targeting miR-148a could be a new therapeutic strategy to protect the bone from damage by prostate cancer metastasis. Previously, miR-181b-5p upregulation was reported to suppress the proliferation, migration and invasion of prostate cancer cells by targeting oncostatin M to modulate RAW264.7 preosteoclast cell osteoclast differentiation (36). Another study previously suggested that hsa-miR-143 in exosomes and trefoil factor 3 are associated with the development of prostate cancer (37). According to the findings aforementioned, prostate tumor cells interact closely with osteoblasts and osteoclasts (38). However, multiple myeloma cell-derived exosomes can directly induce the expression of specific osteoclast markers and modulate the secretion of proteases involved in bone resorption (39). In summary, these aforementioned studies indicated that different kinds of cancer-derived exosomes have different effects on bone; the present study suggested that exosomes from the PC-3 prostate cancer cell line suppressed the differentiation of osteoclasts.

Furthermore, this present study showed that PC-3-derived exosomes significantly repressed the expression of miR-148a in osteoclasts, which is consistent with the finding that downregulating miR-148a attenuated osteoclast differentiation by upregulating MafB (23). Exosomes can deliver cytokines, including receptor activator of nuclear factor- $\kappa \mathrm{B}$ (RANK) and its ligand RANKL and microRNAs, including miR-218 and miR-148a, to modulate osteoclast differentiation during bone resorption (23). Therefore, miR-148a mimics may reduce the progression of PC bone metastases. However, additional 
studies are necessary to investigate the detailed mechanism of miR-148a-mediated regulation of osteoclastogenesis. Further flow cytometry analysis for the characterization of cell surface markers is also required (40). In addition, changes in osteoblast physiology and its surrounding microenvironment following prostate metastasis to be bone would require further analysis.

Overall, the present study aimed to explore the effect of PC-3-derived exosomes on the differentiation of osteoclasts. However, it remains incomplete due to the limitations of not identifying the specific molecules within the exosomes and not determining the effect of osteoblasts on bone homeostasis following PC-3-derived exosomes treatment. In addition, the present study was only performed in vitro. Therefore, experiments in vivo and on clinical specimens would also need to be performed.

In the present study, PC-3-derived exosomes downregulated the expression level of miR-148a and differentiation of osteoclasts by blocking the PI3K/AKT/mTOR signaling pathway. According to these findings, downregulating miR-148a in prostate cancer may serve as a new therapeutic strategy to prevent PC bone metastases. In future studies, the effect of prostate cancer on osteoblast physiology and underlying mechanism would be assessed. Regarding the limitations of the study, since only the PI3K pathway was studied, the next step would be to explore the IKB pathway.

\section{Acknowledgements}

Not applicable.

\section{Funding}

The present study was supported by the National Natural Science Foundation of China (grant no. 81672140) and the Natural Science Foundation of Guangdong Province, China (grant nos. 2018A030313500 and 2017A030313111).

\section{Availability of data and materials}

The datasets used and/or analyzed during the current study are available from the corresponding author on reasonable request.

\section{Authors' contributions}

SN and LZ conceptualized the study and performed the experiments. GT and $\mathrm{KH}$ wrote and edited the manuscript and collected and analyzed data. GT and KH confirmed the authenticity of all the raw data. SQ, YX and YC analyzed data. All authors read and approved the final manuscript.

\section{Ethics approval and consent to participate}

The present study was approved by the Committee of Clinical Ethics of Zhujiang Hospital (Guangzhou, China; approval no. 5574813)). All participants provided written informed consent.

\section{Patient consent for publication}

Not applicable.

\section{Competing interests}

The authors declare they have no competing interests.

\section{References}

1. Taitt HE: Global trends and prostate cancer: A review of incidence, detection, and mortality as influenced by race, ethnicity, and geographic location. Am J Mens Health 12: 1807-1823, 2018.

2. Parkin DM, Bray F, Ferlay J and Pisani P: Global cancer statistics, 2002. CA Cancer J Clin 55: 74-108, 2005.

3. Gilbert SM and McKiernan JM: Epidemiology of male osteoporosis and prostate cancer. Curr Opin Urol 15: 23-27, 2005.

4. Sturge J, Caley MP and Waxman J: Bone metastasis in prostate cancer: Emerging therapeutic strategies. Nat Rev Clin Oncol 8: 357-368, 2011.

5. Wong SK, Mohamad NV, Giaze TR, Chin KY, Mohamed N and Ima-Nirwana S: Prostate cancer and bone metastases: The underlying mechanisms. Int J Mol Sci 20: 2587, 2019.

6. Boukouris S and Mathivanan S: Exosomes in bodily fluids are a highly stable resource of disease biomarkers. Proteomics Clin Appl 9: 358-367, 2015.

7. Mitchell PJ, Welton J, Staffurth J, Court J, Mason MD, Tabi Z and Clayton A: Can urinary exosomes act as treatment response markers in prostate cancer? J Transl Med 7: 4, 2009.

8. Conde-Vancells J, Rodriguez-Suarez E, Embade N, Gil D, Matthiesen R, Valle M, Elortza F, Lu SC, Mato JM and Falcon-Perez JM: Characterization and comprehensive proteome profiling of exosomes secreted by hepatocytes. J Proteome Res 7: 5157-5166, 2008

9. Théry C, Zitvogel L and Amigorena S: Exosomes: Composition, biogenesis and function. Nat Rev Immunol 2: 569-579, 2002.

10. Zhang J, Li S, Li L, Li M, Guo C, Yao J and Mi S: Exosome and exosomal microRNA: Trafficking, sorting, and function. Genomics Proteomics Bioinformatics 13: 17-24, 2015.

11. Png KJ, Halberg N, Yoshida M and Tavazoie SF: A microRNA regulon that mediates endothelial recruitment and metastasis by cancer cells. Nature 481: 190-194, 2011.

12. Lee J, Kwon MH, Kim JA and Rhee WJ: Detection of exosome miRNAs using molecular beacons for diagnosing prostate cancer. Artif Cells Nanomed Biotechnol 46 (Sup3): S52-S63, 2018.

13. Xue M, Zhuo Y and Shan B: MicroRNAs, long noncoding RNAs, and their functions in human disease. Methods Mol Biol 1617: $1-25,2017$.

14. Zeng Z, Li Y, Pan Y, Lan X, Song F, Sun J, Zhou K, Liu X, Ren X, Wang F, et al: Cancer-derived exosomal miR-25-3p promotes pre-metastatic niche formation by inducing vascular permeability and angiogenesis. Nat Commun 9: 5395, 2018.

15. Hsu YL, Hung JY, Chang WA, Lin YS, Pan YC, Tsai PH, Wu CY and Kuo PL: Hypoxic lung cancer-secreted exosomal miR-23a increased angiogenesis and vascular permeability by targeting prolyl hydroxylase and tight junction protein $\mathrm{ZO}-1$. Oncogene 36 : 4929-4942, 2017.

16. Bartel DP: MicroRNAs: Genomics, biogenesis, mechanism, and function. Cell 116: 281-297, 2004.

17. Mitchell PS, Parkin RK, Kroh EM, Fritz BR, Wyman SK, Pogosova-Agadjanyan EL, Peterson A, Noteboom J, O'Briant KC, Allen A, et al: Circulating microRNAs as stable blood-based markers for cancer detection. Proc Natl Acad Sci USA 105: 10513-10518, 2008.

18. Landgraf P, Rusu M, Sheridan R, Sewer A, Iovino N, Aravin A, Pfeffer S, Rice A, Kamphorst AO, Landthaler M, et al: A mammalian microRNA expression atlas based on small RNA library sequencing. Cell 129: 1401-1414, 2007.

19. Bellavia D, Salamanna F, Raimondi L, De Luca A, Carina V, Costa V, Alessandro R, Fini M and Giavaresi G: Deregulated miRNAs in osteoporosis: Effects in bone metastasis. Cell Mol Life Sci 76: 3723-3744, 2019.

20. Seeliger C, Karpinski K, Haug AT, Vester H, Schmitt A, Bauer JS and van Griensven M: Five freely circulating miRNAs and bone tissue miRNAs Are associated with osteoporotic fractures. J Bone Miner Res 29: 1718-1728, 2014.

21. Bedene A, Mencej Bedrač S, Ješe L, Marc J, Vrtačnik P, Preželj J, Kocjan T, Kranjc T and Ostanek B: MiR-148a the epigenetic regulator of bone homeostasis is increased in plasma of osteoporotic postmenopausal women. Wien Klin Wochenschr 128 (Suppl 7): S519-S526, 2016. 
22. Kelch S, Balmayor ER, Seeliger C, Vester H, Kirschke JS and van Griensven M: miRNAs in bone tissue correlate to bone mineral density and circulating miRNAs are gender independent in osteoporotic patients. Sci Rep 7: 15861, 2017.

23. Cheng P, Chen C, He HB, Hu R, Zhou HD, Xie H, Zhu W, Dai RC, Wu XP, Liao EY and Luo XH: miR-148a regulates osteoclastogenesis by targeting V-maf musculoaponeurotic fibrosarcoma oncogene homolog B. J Bone Miner Res 28: 1180-1190, 2013.

24. Chen H, Zhou L, Wu X, Li R, Wen J, Sha J and Wen X: The $\mathrm{PI} 3 \mathrm{~K} / \mathrm{AKT}$ pathway in the pathogenesis of prostate cancer. Front Biosci (Landmark Ed) 21: 1084-1091, 2016.

25. Mayer IA and Arteaga CL: The PI3K/AKT pathway as a target for cancer treatment. Annu Rev Med 67: 11-28, 2016.

26. Edlind MP and Hsieh AC: PI3K-AKT-mTOR signaling in prostate cancer progression and androgen deprivation therapy resistance. Asian J Androl 16: 378-386, 2014.

27. Zhu W, Hu X, Xu J, Cheng Y, Shao Y and Peng Y: Effect of PI3K/Akt signaling pathway on the process of prostate cancer metastasis to bone. Cell Biochem Biophys 72: 171-177, 2015.

28. Tang LA, Dixon BN, Maples KT, Poppiti KM and Peterson TJ: Current and investigational agents targeting the phosphoinositide 3-kinase pathway. Pharmacotherapy 38: 1058-1067, 2018.

29. Théry C, Amigorena S, Raposo G and Clayton A: Isolation and characterization of exosomes from cell culture supernatants and biological fluids. Curr Protoc Cell Biol Chapter 3: Unit 3.22, 2006.

30. Skog J, Würdinger T, van Rijn S, Meijer DH, Gainche L, Sena-Esteves M, Curry WT Jr, Carter BS, Krichevsky AM and Breakefield XO: Glioblastoma microvesicles transport RNA and proteins that promote tumour growth and provide diagnostic biomarkers. Nat Cell Biol 10: 1470-1476, 2008.

31. Urabe F, Kosaka N, Kimura T, Egawa S and Ochiya T: Extracellular vesicles: Toward a clinical application in urological cancer treatment. Int J Urol 25: 533-543, 2018.

32. Schmittgen T and Livak K: Analyzing real-time PCR data by the comparative C(T) method. Nat Protoc 3: 1101-1108, 2008.
33. Janssen JC, Woythal N, Meißner S, Prasad V, Brenner W, Diederichs G, Hamm B and Makowski MR: $\left[{ }^{68} \mathrm{Ga}\right]$ PSMA-HBED-CC uptake in osteolytic, osteoblastic, and bone marrow metastases of prostate cancer patients. Mol Imaging Biol 19: 933-943, 2017.

34. Fang J and Xu Q: Differences of osteoblastic bone metastases and osteolytic bone metastases in clinical features and molecular characteristics. Clin Transl Oncol 17: 173-179, 2015.

35. Torrealba N, Rodriguez-Berriguete G, Fraile B, Olmedilla G, Martínez-Onsurbe P, Sánchez-Chapado M, Paniagua R and Royuela M: PI3K pathway and Bcl-2 family. Clinicopathological features in prostate cancer. Aging Male 21: 211-222, 2018.

36. Shao B, Fu X, Yu Y and Yang D: Regulatory effects of miRNA-181a on FasL expression in bone marrow mesenchymal stem cells and its effect on CD4+T lymphocyte apoptosis in estrogen deficiency-induced osteoporosis. Mol Med Rep 18: 920-930, 2018

37. Che Y, Shi X, Shi Y, Jiang X, Ai Q, Shi Y, Gong F and Jiang W: Exosomes derived from miR-143-overexpressing MSCs inhibit cell migration and invasion in human prostate cancer by downregulating TFF3. Mol Ther Nucleic Acids 18: 232-244, 2019.

38. Fornetti J, Welm AL and Stewart SA: Understanding the bone in cancer metastasis. J Bone Miner Res 33: 2099-2113, 2018.

39. Raimondi L, De Luca A, Amodio N, Manno M, Raccosta S, Taverna S, Bellavia D, Naselli F, Fontana S, Schillaci O, et al: Involvement of multiple myeloma cell-derived exosomes in osteoclast differentiation. Oncotarget 6: 13772-13789, 2015.

40. Fathi E, Valipour B, Sanaat Z, Nozad Charoudeh H and Farahzadi R: Interleukin- $6,-8$, and TGF- $\beta$ secreted from mesenchymal stem cells show functional role in reduction of telomerase activity of leukemia cell via $\mathrm{Wnt} 5 \mathrm{a} / \beta$-catenin and P53 pathways. Adv Pharm Bull 10: 307-314, 2020. International (CC BY-NC-ND 4.0) License. 